\title{
One-Stage Hybrid Procedure for Patent Ductus Arteriosus Combined with Other Cardiac Anomalies in Adults
}

\author{
Hong-Chang Guo, MD,${ }^{1}$ Yang Wang, MD,${ }^{1,3}$ Wen-Hui Wu, MD,${ }^{2}$ Yong-Qiang Lai, $\mathrm{MD}^{1}$ \\ ${ }^{1}$ Department of Cardiac Surgery and 2Intervention Department, Beijing Anzhen Hospital, Capital Medical University, Beijing, China; \\ ${ }^{3}$ Department of Cardiac Surgery, Affiliated Hospital of HeBei University, Baoding, Hebei, China
}

\section{ABSTRACT}

Patients with patent ductus arteriosus (PDA) are usually detected and treated during childhood, however, some are not detected until adulthood. In addition, heart failure, severe pulmonary hypertension, and calcification of the ductus may also exist. Transcatheter occlusion has become a widely used technique for the closure of small to moderate sized PDAs since Cambier first reported use of Gianturco coils for transcatheter closure of PDA in 1992 [Cambier 1992]. In the case studies reported here, the one-stage hybrid procedure for PDA combined with other cardiac anomalies in adults was applied with satisfactory results.

\section{CASE REPORTS}

\section{Case One}

A 43-year-old woman with exertional chest discomfort and dyspnea was admitted. She had a history of stroke with no sequelae. Physical examination revealed persistent murmur along the left sternal border and a normal $\mathrm{SaO} 2$ (98.2\%). Echocardiography revealed a tubular-type patent ductus arteriosus (PDA) with bidirectional shunt, left-sided cardiomegaly, and pulmonary hypertension. A $24 \mathrm{~mm} \times 49 \mathrm{~mm}$ left atrial myxoma was detected. Cardiac catheterization demonstrated a $10 \mathrm{~mm}$ tubular PDA and pulmonary artery hypertension $(50 / 28 \mathrm{mmHg})$. An LT-PDA-06-08 Cera PDA occluder (Lifetech Scientific Corporation, Shenzhen, China) was deployed by using a 6-F sheath through the right femoral vein. Complete closure of the ductus was indicated immediately with angiography. This patient underwent open-heart surgery for myxoma resection with normothermic cardiopulmonary bypass one day later. Complete resection was obtained through atrium septal incision. She had an uneventful recovery, and remained well during a 12-month follow-up.

\section{Case Two}

A 35-year-old woman was referred to our institution with a diagnosis of PDA and ventricular septal defect (VSD).

Received March 19, 2017; received in revised form April 13, 2018; accepted April 16, 2018.

Correspondence: Yong-Qiang Lai, MD, Department of Cardiac Surgery, Beijing Anzhen Hospital, Beijing 100029, China; +86-10-64456876 (e-mail: yongqianglai@yahoo.com).
Congenital heart disease had been diagnosed in her childhood. There were no obvious symptoms until the preceding year. Echocardiography demonstrated a tubular-type PDA with a bidirectional shunt, a perimembrane VSD about $12 \mathrm{~mm}$ in diameter, and pulmonary artery hypertension. Cardiac catheterization demonstrated a $6 \mathrm{~mm}$ tubular PDA and pulmonary artery hypertension $(62 / 31 \mathrm{mmHg})$. The PDA was closed with a Cardi-O-Fix PDA occluder (Starway medical technology, INC, Beijing, China), and the VSD was repaired using a dacron patch with normothermic cardiopulmonary bypass one day later. The patient recovered well during a 12-month follow-up.

\section{DISCUSSION}

Diagnosis and treatment may be delayed in some adult patients with PDA due to a lack of symptoms. Closure of the PDA is indicated in adults in order to prevent the hemodynamic changes of a left-to-right shunt, including aneurysm formation, heart failure, pulmonary hypertension, and endocarditis. One-stage operation remains the most common choice for adult PDA patients with other cardiac anomalies.

Because of the fragility of the aorta and the aneurysmal change of the ductus, surgical closure of PDA in adult patients carries a higher risk. When pulmonary hypertension is present, the risk present during surgical repair of PDA increases dramatically, especially when the transpulmonary closure technique is used with hypothermic circulatory arrest (DHCA) [Toda 2000]. Disadvantages of DHCA include increased cardiopulmonary bypass (CPB) time, edema formation, coagulopathy, and influence on multi-organ functions. In addition, spinal cord ischemic reperfusion injury is a fatal complication during DHCA [Drinkwater 2010].

Transcatheter closure with an umbrella or spring coil has proved to be an effective and safe treatment. When a large PDA and severe pulmonary hypertension are present, dislodgment of the umbrella is a serious complication [Yan 2007]. In this circumstance, thoracic endovascular aortic repair may be a therapeutic alternative for adult patients with large PDA and pulmonary hypertension [Lai 2008]. Both patients in this report had associated pulmonary hypertension, and the one-stage hybrid procedure was applied. PDA was occluded during cardiac catheterization examination. Associated cardiac anomalies were corrected with normothemic cardiopulmonary bypass. Because there was no hybrid operating room, the other procedures were performed the 
next day. This technique can avoid the surgical risks of PDA repair and the use of DHCA, and decrease the occurrence of some serious complications. Patients recovered uneventfully and persisted well within a short-term follow-up.

Our preliminary experience demonstrated that one-stage hybrid procedure may be a safe and easy alternative for adult PDA patients with other cardiac anomalies.

\section{REFERENCES}

Cambier PA, Kirby WC, Wortham DC, et al. 1992. Percutaneous closure of the small (less than $2.5 \mathrm{~mm}$ ) patent ductus arteriosus using coil embolization. Am J Cardiol 69:815-16.
Drinkwater SL, Goebells A, Haydar A, et al. 2010. The incidence of spinal cord ischaemia following thoracic and thoracoabdominal aortic endovascular intervention. Eur J Vasc Endovasc Surg 40:729-35.

Lai YQ, Xu SD, Li ZZ, et al. 2008. Thoracic endovascular aortic repair of adult patent ductus arteriosus with pulmonary hypertension. J Thorac Cardiovasc Surg 135:699-701.

Toda R, Moriyama Y, Yamashita M, et al. 2000. Operation for adult patent ductus arteriosus using cardiopulmonary bypass. Ann Thorac Surg 70:1935-8

Yan C, Zhao S, Jiang S, et al. 2007. Transcatheter closure of patent ductus arteriosus with severe pulmonary arterial hypertension in adults. Heart 93:514-18. 